\title{
Pränatales Screening auf Trisomie 21: Einsatz des Praena-Tests
}

\begin{abstract}
Hintergrund
Seit Juli 2012 ist in der Schweiz ein neuer Test zur vorgeburtlichen Diagnostik erhältlich. Der sog. PraenaTest der Firma Lifecodexx ist ein nicht-invasiver Test zur Detektion von Trisomie 21. In einer mütterlichen Blutprobe kann festgestellt werden, ob der Anteil an im Plasma zirkulierender DNA des Chromosoms 21 als Folge einer Trisomie 21 des Fötus erhöht ist. Die Schweizerische Gesellschaft für Gynäkologie und Geburtshilfe empfiehlt, den Test nur bei Patientinnen mit einem erhöhten Risiko für Trisomie 21 durchzuführen. Für eine generelle Anwendung wurde der Test bisher nicht validiert. Grundsätzlich gilt, dass falsch positive Resultate umso häufiger sind, je geringer das Risiko für eine Trisomie 21 vor dem Test ist. Bei positivem Testergebnis wird in jedem Fall empfohlen, die Diagnose durch eine Amniozentese zu überprüfen, bevor eine Entscheidung über einen Abbruch der Schwangerschaft getroffen wird (www.sggg.ch).

In den Medien wurde der Praena-Test kontrovers diskutiert; die ZEK nimmt nachfolgend Stellung zu den ethisch relevanten Aspekten.
\end{abstract}

\section{Ethisch relevante Aspekte}

Die Angebote der vorgeburtlichen Diagnostik genetischer Anomalien, die nach Empfehlung der Schweizerischen Gesellschaft für Gynäkologie und Geburtshilfe schon bei der ersten Konsultation der werdenden Mutter besprochen werden sollen, können für das Paar, das sich ein möglichst gesundes Kind wünscht, eine Erweiterung der Optionen zur Selbstbestimmung darstellen. Die zunehmende Verfügbarkeit von neuen technischen Möglichkeiten im Bereich der vorgeburtlichen Diagnostik, die auf den ersten Blick risikolos erscheinen, birgt aber auch die Gefahr in sich, dass werdende Mütter beziehungsweise Eltern unreflektiert in Tests einwilligen, über deren Reichweite sie sich nicht bewusst sind. Fehlende Invasivität und hohe Chancen für ein beruhigendes Resultat erleichtern eine Einwilligung mit dem Vorbehalt, belastende ethische Entscheidungen gegebenenfalls später treffen zu können. Wenn dann jedoch der Fall eines hohen Risikos oder der Gewissheit einer Trisomie 21 eintritt, muss unter grossem Zeitdruck und emotionaler Belastung entschieden werden. Zudem kann die Entscheidungsfreiheit eingeschränkt sein, wenn die Möglichkeit, die Geburt eines Kindes mit Trisomie 21 zu verhindern, als sozialer Druck erlebt wird. Aus ethischer Sicht ist deshalb zu fordern, dass der Entscheid mit Zeit und in Ruhe getroffen werden kann und Erwartungshaltun- gen, mit denen Frauen respektive Paare konfrontiert sind, reflektiert werden. Damit Entscheide zur pränatalen Diagnostik vollständig informiert und wohlüberlegt getroffen werden können, sollten die Konsequenzen der Geburt eines Kindes mit Trisomie 21, bzw. des späten Abbruchs einer primär erwünschten Schwangerschaft für den eigenen Lebensentwurf so weit wie möglich absehbar sein. Dafür sind Kenntnisse über das Leben von Familien mit einem Kind mit Trisomie 21 und Orientierung über Unterstützungsangebote wichtig. Jede schwangere Frau respektive jedes Paar ist darüber aufzuklären, dass die Durchführung eines vorgeburtlichen genetischen Screenings in der Regel keine Vorsorgeuntersuchung zum Wohl des Kindes ist, sondern einer Auswahlentscheidung zur Verhinderung eines Kindes mit bestimmten genetischen Merkmalen dient.

In einer am Grundsatz der Gerechtigkeit orientierten Gesellschaft hat die gleichberechtigte Teilhabe (Inklusion) aller Mitglieder am gesellschaftlichen Leben einen hohen Stellenwert. Dieser Anspruch ist gesetzlich verankert und muss laufend umgesetzt werden. Die parallel zu diesen Bestrebungen beobachtbare gesellschaftliche Tendenz, Behinderung so weit wie möglich systematisch auszuschliessen, wird von vielen Betroffenen und ihren Angehörigen als Bedrohung erlebt. Es ist deshalb essentiell, dass der rechtlich gewährleistete Schutz von Menschen mit genetischen Auffälligkeiten erhalten und weiter ausgebaut wird.

\section{Empfehlungen der ZEK}

Gestützt auf die obigen Ausführungen empfiehlt die ZEK die Beachtung folgender Punkte:

- Die Beratung der Schwangeren im Hinblick auf die pränatale Diagnostik ist so zu gestalten, dass eine möglichst autonome Entscheidung sichergestellt ist.

- Kenntnisse zum pränatalen Screening und seiner ethischen Implikation sollten so früh wie möglich vermittelt werden (z.B. im Rahmen der Schulbildung).

- Die Unterstützung und Inklusion von Menschen, die mit Trisomie 21 leben, und ihren Familien ist zu verbessern.

- Der rechtlich gewährleistete Schutz von Menschen mit genetischen Auffälligkeiten vor Diskriminierung ist mindestens aufrechtzuerhalten.

Von der Zentralen Ethikkommission der SAMW am 26. Oktober 2012 genehmigt. 